\title{
Can Electric Field be a Descriptor of Catalytic Activity? A Case Study on Chorismate Mutase
}

\author{
Shakir Ali Siddiqui ${ }^{\mathrm{a}}$ and Kshatresh Dutta Dubey, ${ }^{\mathrm{a}, \mathrm{b} *}$
}

a. Department of Chemistry, School of Natural Sciences, Shiv Nadar University Delhi-NCR, 201314, India.

b. Center for Informatics, Department of Chemistry, School of Natural Sciences, Shiv Nadar University Delhi-NCR, 201314, India.

\begin{abstract}
:
The current theoretical perception of enzymatic activity is highly reliant on the determination of activation energy of the reactions which is often calculated using a computational demanding quantum mechanical calculation. With the ever-increasing use of bioengineering techniques that produce too many variants of the same enzyme, a fast and accurate way to study the relative efficiency of enzymes is a need of time. Here, we propose the electric field (EF) of the enzyme along the reaction axis as a descriptor for the enzymatic activity using an example of Chorismate Mutase in its native and several variants (R90A, R90G, and R90K/C88S). The study shows a linear correlation between the calculated enzymatic EF and enzymatic activity of all complexes. The MD simulations of the Michaelis complex and the transition state analog (TSA) show a stabilizing force on TSA due to the enzymatic EF. The QM/MM and QM-only DFT calculations with the presence of External Electric Field (EEF) oriented along the reaction axis show that the electric field can increase the dipole moment of the TS, thereby, stabilizing it and thus lowers the activation energy.
\end{abstract}




\section{Introduction:}

A target-based enzyme design is an ultimate goal for the bioengineers as it gives a greener and sustainable approach for synthesis of desired products [1-5]. This goal is now possible by mutating some key strategic residues of the active site using directed evolution method, [6-10] which is efficient, but at the same time, is random. Therefore, a comprehensive understanding of the molecular mechanism that confers better activity could improve such design protocols as well as could also guide the development of smarter mutagenesis and screening strategies. But, unlike a protein-drug interactions where the binding information of the drug using lock-key concept could be enough [11-14], understanding the molecular mechanism of the enzyme catalysis is somewhat tricky. An enzymatic reaction, according to Warshel, can involve two components [15-17]: the first component is the entropic contribution to the binding free energy for bringing the reactive fragment into the catalytic site which is equivalent to dissociation constant, and the second fragment is the free energy needed to reach the TS from the reactant geometry and is equivalent to activation energy. Fortunately, modern methods like MD simulations [13, 18-20] can deal the information related to the first component (binding stability) while hybrid QM/MM calculations can reveal the second component i.e., activation energy [21-24]. A combination of these two methods, therefore, provides a crucial lead to design more effective and powerful enzyme for modern use. More recently, with ever increased rate, the external electric field (EEF) is being used to harness the catalytic activity of many chemical reactions [25-35]. In fact, Nature routinely harnesses pre-oriented local-electric fields (LEF) for the electrostatic catalysis observed in enzymes. Therefore, the quantification of electric field in the enzyme system using computational tools could be an add-on to the rational based enzyme designing. In the present study, we show how the quantification of electric field (EF) along the axis of reaction could be used as a descriptor for the enzyme activity. 
In the present study, we quantify the local electric field produced by Chorismate Mutase and calculated the reactivity of the enzyme in presence of oriented electric field using hybrid QM/MM calculations. Chorismate Mutase is an enzyme formally known to catalyse the Claisen rearrangement where (-) chorismate is converted into prephenate as shown in Scheme 1, and hence presents a rare example of enzyme catalysed pericyclic process [36-42].

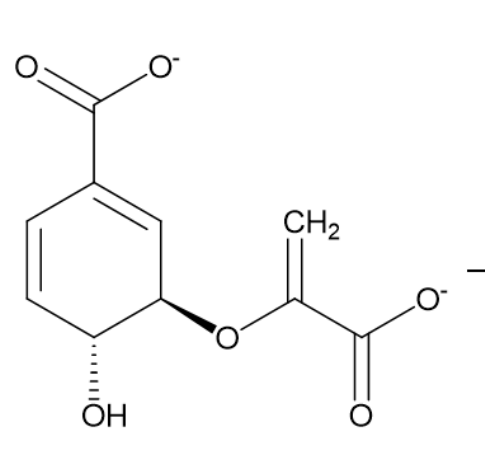

Chorismate

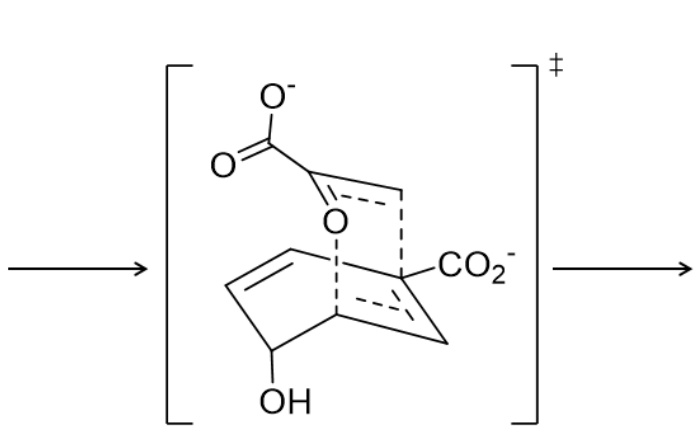

Transition State

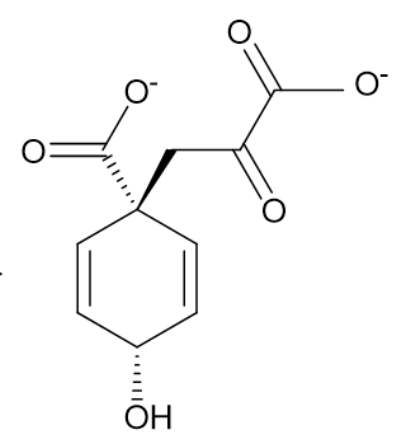

Prephenate

Scheme 1. Schematic Representation of Conversion of Chorismate to prephenate.

Our choice of Chorismate Mutase (CM) as a model system to study the electric field effect is based mainly due to its peculiar characteristic to catalyse reaction using electrostatic environment created by the protein without involvement of any covalent bonding, and therefore, the effect of electric field could be more prominent in CM. Furthermore, sufficient experimental studies for WT and its several variants are also available which can be used to benchmark the computational findings [38-39,43,44]. In the same context, Hilvert group, using experimental gene engineering of the active site residues, emphasized the role of positively charged residue e.g., R90/K90 to stabilize the transition state (TS) of the charismatic rearrangement. This finding was further justified by site directed mutagenesis studies performed by the same group in a separate study where they mutated R90 with Alanine and Glycine, and found that the catalytic activity was decreased by 5 -fold relative to WT complex. The activity was again restored when a Lysine residue 
was reinstated at the same position. These findings clearly confirm a crucial role of the electrostatic environment in the catalysis. In the present study, therefore, we have used WT chorismate mutase along with its variant to establish a linear correlation between enzymatic electric field and its catalytic efficiency using MD simulations, and hybrid QM/MM calculations. Using an example of Chorismate Mutase, we show that Electric field can be used as an efficient descriptor for the enzymatic activity.

\section{Computational Details:}

In the present study, we have used MD simulations for the conformational and binding stabilities of the $\mathrm{CM}$ and its variant, $\mathrm{QM} / \mathrm{MM}$ calculations for the mechanism of chorismate to prephenate conversion, and quantification of electric field for WT and its mutant complexes. The details of each method are described below.

\section{1. System Setup:}

The initial coordinates for the WT CM complex were taken from the crystal structure of Bacillus subtilis chorismate mutase enzyme bound with Bartlett's inhibitor (a transition state analogue inhibitor) from the protein data bank (PDB code: 2CHT) [45]. The imported structure contains several missing hydrogen atoms which were added by Leap module of Amber 20. Three different variants of the Bacillus subtilis chorismate mutase enzyme, i.e., R90A, R90G and R90K/C88S variants were produced by mutating Arg90 residues using pdb4amber module of Amber 20 . The parameters for the TSA inhibitor and substrate chorismate were generated by using antechamber module of Amber20 for GAFF2 parameters for a QM optimized geometry at HF/6-31G(d,p) level of theory. Thereafter, all complexes were solvated in a truncated octahedron box of TIP3P water molecules with $12 \AA$ cutoff from the protein boundary [46]. An appropriate number of Na+ ions 
were added to neutralize the total charge of the system. Since crystal structure of the CM was with TSA, we used the same active site conformation to dock chorismate substrate using Autodock Vina implemented in UCSF Chimera [47].

\subsection{Simulations:}

After the initial setup of the complexes in WT and its mutants, systems were minimized in two steps for removing the bad contacts. In the step 1, only solvent molecules were minimized using 5000 steps of steepest descent followed by a 5000 steps of conjugate gradient minimizer. In the step 2, all the complexes were minimized without any constraints using the same minimizer as in step 1. All systems were then gently annealed from 10 to $300 \mathrm{~K}$ under NVT ensemble for 50 ps with a weak restraint of $5 \mathrm{kcal} / \mathrm{mol} / \AA^{2}$. Subsequently a density equilibrium was performed using NPT ensemble at targeted temperature of $300 \mathrm{~K}$ and pressure 1 atm for $1 \mathrm{~ns}$ using the Langevinthermostat and Berendsen-barostat, with a weak restraint of $1 \mathrm{kcal} / \mathrm{mol} / \AA^{2}[48,49]$. Thereafter, we removed all the restrains and the systems were further equilibrated for $3 \mathrm{~ns}$ followed by $50 \mathrm{~ns}$ of production simulations for each system with TSA and $100 \mathrm{~ns}$ for Michaelis Complex. The covalent

bonds that contain hydrogens were constrained using SHAKE algorithm, and a particle mesh Ewald (PME) was utilized to treat long-range electrostatic interactions during all the MD simulations [50,51]. An integration step of 2 fs was used during the entire simulation. All the MD simulations were performed with the GPU version of the Amber 20 package [52].

\subsection{QM/MM Calculations:}

The reaction mechanism was investigated by use of the QM/MM calculations for the representative snapshots from the MD trajectories for each system. 
QM regions included R90, E78 and entire substrate molecule, the coordinates for the QM region can be found in SI. All protein residues and water molecules within $8 \AA$ of the substrate were included in the 'active region' of the QM/MM calculations. The atoms in the 'active region' interact with the QM atoms through electrostatic and van der Waals interactions and the corresponding polarization effects were considered in the subsequent QM/MM calculations. All QM/MM calculations were performed with ChemShell, by combining Turbomole for the QM part, and DL_POLY for the MM part [53-56]. The MM region was described using the Amber ff14SB force field, and the electronic embedding scheme was used to account for the polarizing effect of the enzyme environment on the QM region [57]. The QM/MM boundary was treated using hydrogen link atoms with the charge-shift model [54].

During QM/MM geometry optimizations, the QM region was treated using the hybrid B3LYP functional with def2-SVP basis set [58]. The TS optimization was performed by dimer method included in the Chemshell package [53].

\subsection{QM only DFT Calculations:}

The PES scanning for the interconversion reaction of chorismate into the prephenate in the gasphase was determined by the Gaussian09 [59]. For geometry optimization and frequency calculations, we used the hybrid B3LYP/def2-SVP level of theory [58]. The effects of EEFs were also studied using Gaussian09. A range of electric field strengths $\left(\mathrm{F}_{\mathrm{y}}\right)$ was explored while allowing to be oriented along the y-axis i.e., the reaction axis, along which the bonds are being broken and formed. For the geometry optimization in the presence of EEF, we used internal coordinates to avoid the misalignment of the EEF during the variation of the geometry.

\section{Results \& Discussion:}




\subsection{MD simulations of Michaelis Complex and Transition state analog (TSA) in WT}

\section{Chorismate Mutase:}

We performed MD simulation of the WT complex of chorismate mutase with substrate for Michaelis Complex (MC) and Transition State Analog (TSA) to study the effect of local electric field produced by native enzyme on the stability of the reactant and transition state. Though the active site of the enzyme is surrounded by many charge residues such as R7, E78, R90, Y108, K60 and R63, but the reactant shows significant degree of flexibility during the simulations. Figure 1 shows three representative snapshots from the MD simulations of MC at different time scale. It is evident from the figure that during simulation substrate loses several interactions with
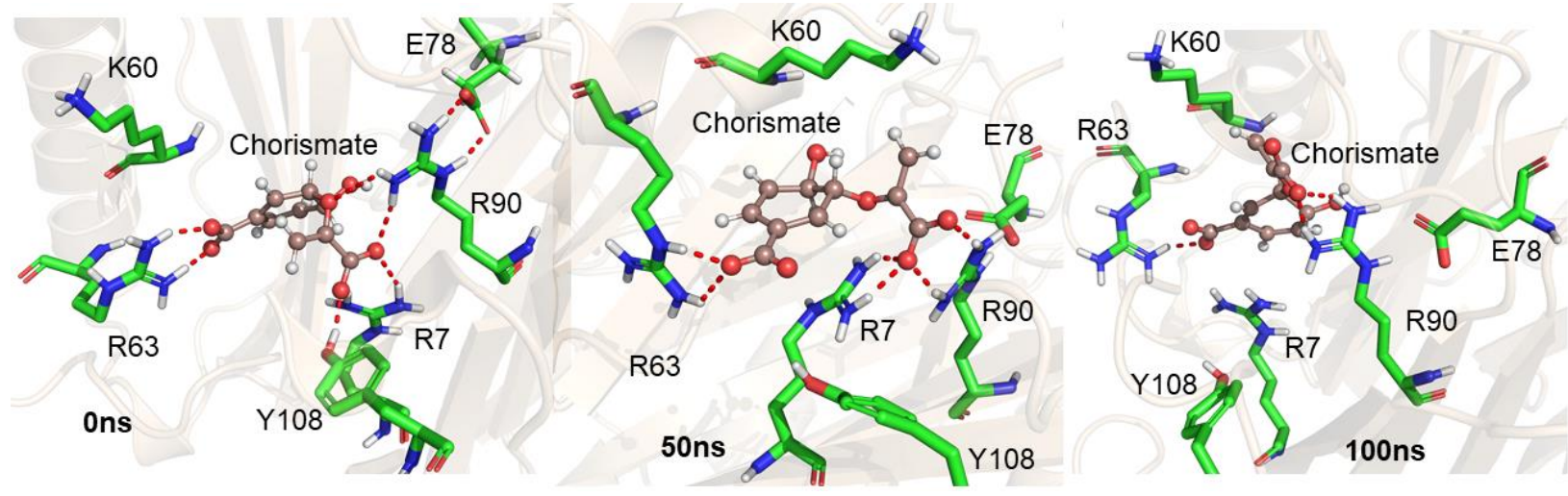

Figure 1. Representative snapshots of MD Trajectory of Michaelis Complex in WT Chorismate Mutase showing the interaction of the substrate with the key residues at different time scale.

key residues such as R7 and Y108 which can be anticipated by the increasing distances of these residues with the substrate continuously with simulation time. To further quantify the effect of the charged residue on the reactant we calculated the total change in binding enthalpy using MMPBSA method. The calculation shows $\Delta \mathrm{H}_{\text {binding }}$ as $-31.0 \mathrm{kcal} / \mathrm{mol}$ which is mainly dominated by strong and favorable interactions by R7, R90, K60 and R63. 
Unlike MC, the simulation of the transition state analog (TSA) with WT shows a stable complex during entire simulations. A consistent interaction is shown with R7 and Tyr108
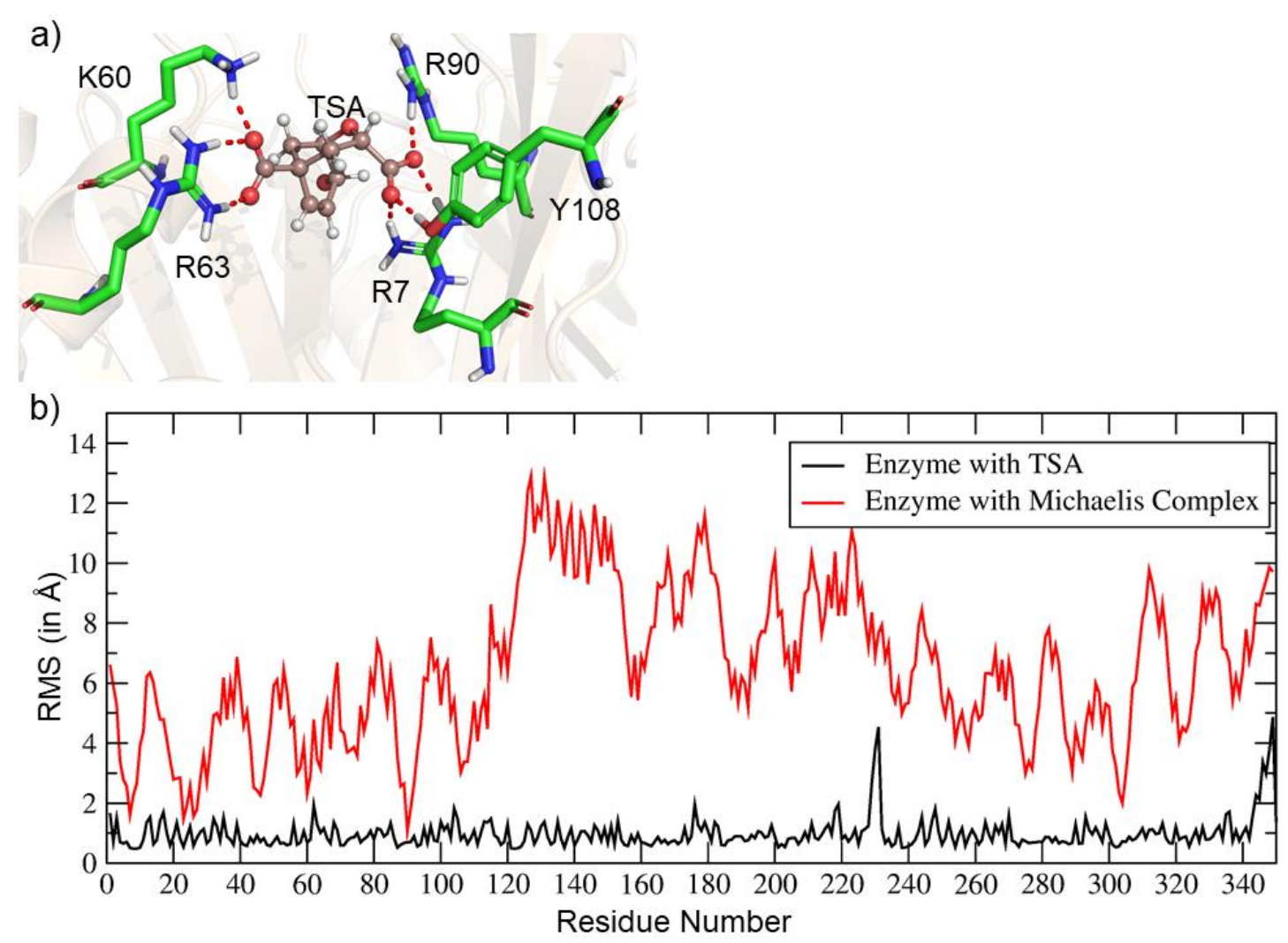

c)

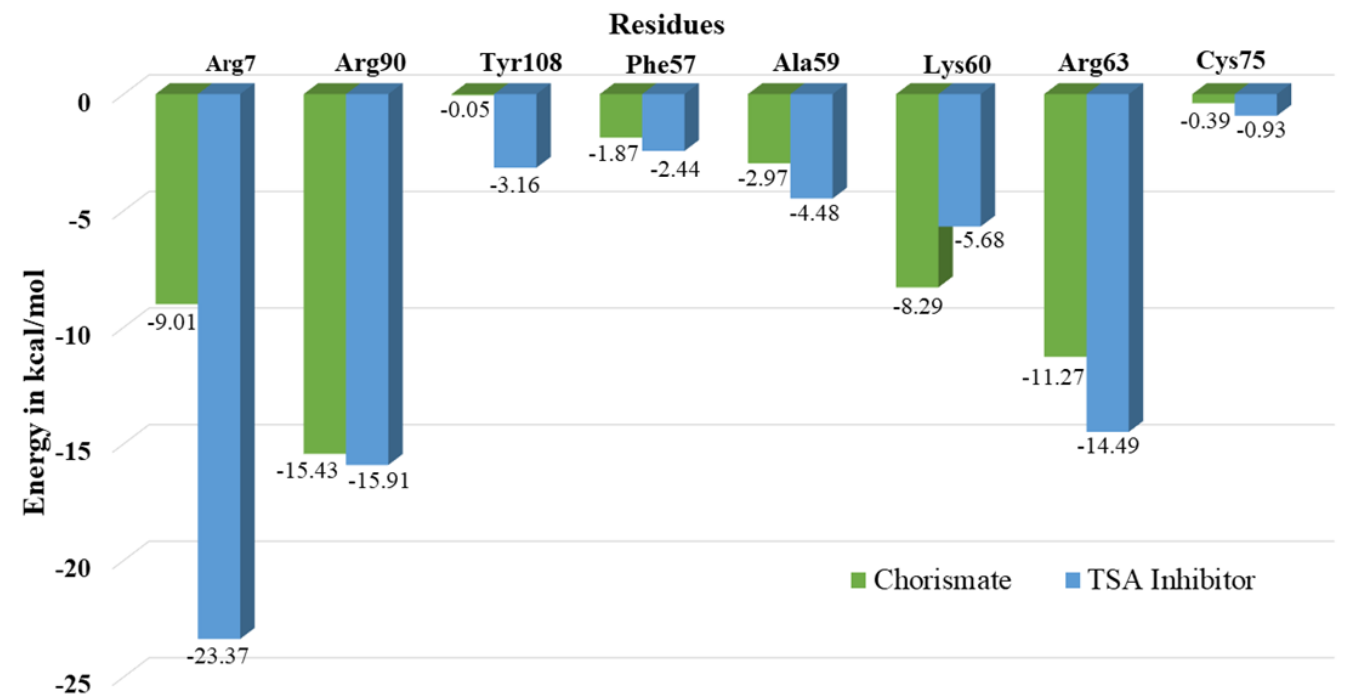


Figure 2. a) Key Residues of WT CM interacting with the TSA. b) The plot showing the RMSF values in $\AA$ for Transition state analog (TSA) and Michaelis Complex in WT Chorismate Mutase. c) A comparative residue interaction map between Chorismate and the TSA inhibitor for the most populated trajectories.

during entire simulation as shown in Figure 2a. The root means square fluctuations both complexes (MC and TSA) are shown in Figure 2b which clearly indicates TSA as less flexible and hence more stable during simulations. It is further substantiated by a comparison of residue interaction network for MC and TSA as shown in Figure 2c which also shows more favorable interactions relative to MC. Therefore, in nutshell, the chorismate mutase has evolved in such a manner that the local electric field produced by enzyme reduces the entropic penalties of the transition state analog (TSA) and thus lowers the overall barrier of reactions.

\subsection{The Mechanism of reaction by Chorismate Mutase by QM/MM Calculations}

So far, using MD simulation of the Michaelis Complex and TSA, we have seen that the local electric field produced by enzyme provides an entropic favor in transition state. However, we complemented our study with QM/MM calculations and a gas-phase Density Functional Calculations to elaborate the role of electrostatics on the activation energy for chorismate to

prephenate. The gas-phase density functional calculations show that conversion of chorismate to prephenate is an energetically demanding process and the energy barrier for this process (in absence of enzymatic system) is $32.90 \mathrm{kcal} / \mathrm{mol}$ (Figure 3). 


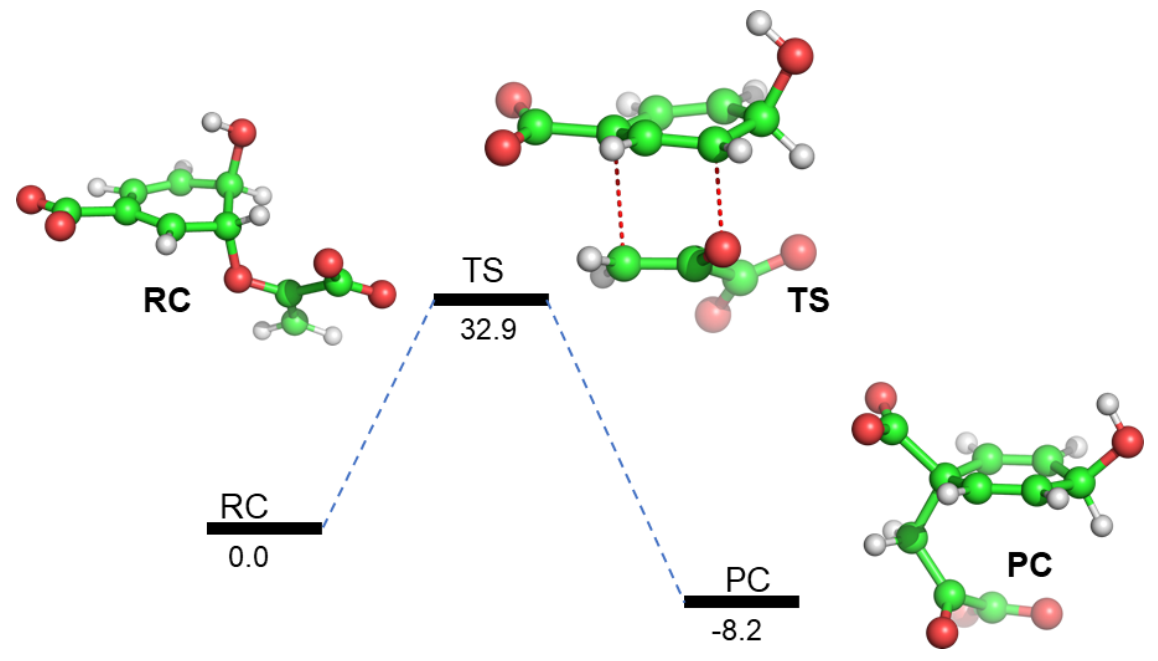

Figure 3. The B3LYP/Def2-SVP gas-phase energy profile diagram for the interconversion of chorismate to prephenate. The energies are reported in $\mathrm{kcal} / \mathrm{mol}$ relative to the $\mathrm{RC}$.

Interestingly, when we included the enzyme environment using QM/MM calculations the barrier of the same reaction decreased by a significant amount (see Figure 4 for QM/MM reaction profile). The QM/MM calculation was started by considering a representative snapshot from

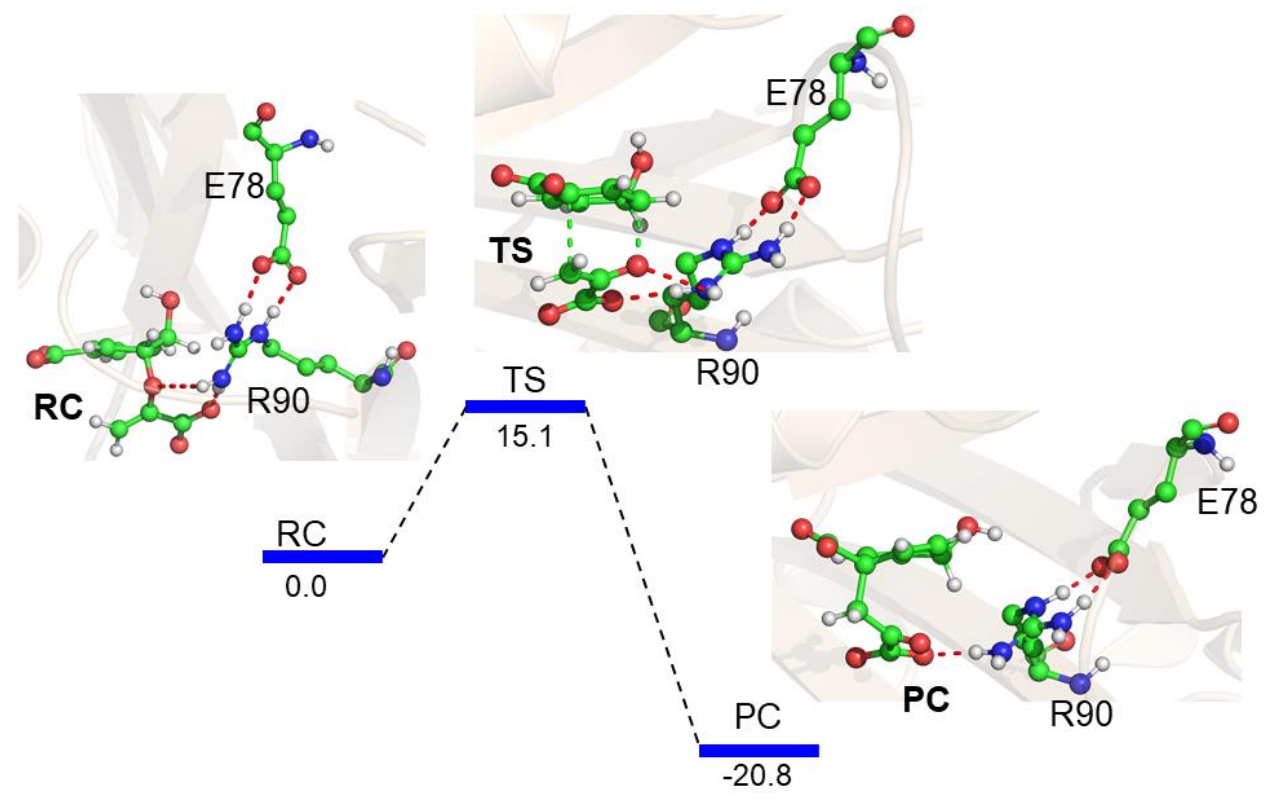

Figure 4. QM/MM/B3LYP/Def2-SVP energy profile for the rearrangement of chorismate into prephenate, with its characteristic species indicated as follows: reactant complex (RC), transition state (TS) and product complex (PC). The energies (relative to RC) are shown in $\mathrm{kcal} / \mathrm{mol}$. 
the MD simulations and a subsequent optimization of the Michaelis Complex. A relaxed potential energy surface (PES) scanning was performed for the conversion of chorismate to prephenate. The energy profile shows a feasible reaction with a TS barrier of $15.1 \mathrm{kcal} / \mathrm{mol}$ for this transformation when it occurs in the enzymatic environment. In addition, the product prephenate is more exothermic relative to the gas-phase reaction.

It is noteworthy that none of the protein residues of the CM participate in the reaction, therefore, how does the enzyme catalyze the reaction so efficiently? In order to explore the possible cause, we further quantified the LEF produced by the enzyme using TITAN program.

\subsection{Quantification of Electric Field by Enzyme and its Catalytic Effect}

We quantified the electric field exerted by enzyme along $\mathrm{C}-\mathrm{O}$ bond (see Figure $5 \mathrm{a}$ and $5 \mathrm{~b}$ ) of the substrate to probe the electric field effect. For doing so, we used the optimized structures from the QM/MM calculations as mentioned above. Our calculations show a net electric field of $-\mathbf{0 . 2}$ V/Å directed along of reaction centre in the the $\mathrm{C}-\mathrm{O}$ axis at the site absence of solvent molecules (LEF of enzyme alone) while it is - $\mathbf{0 . 5 3}$ V/A in presence of solvent molecules (see Figure 5a and 5b).
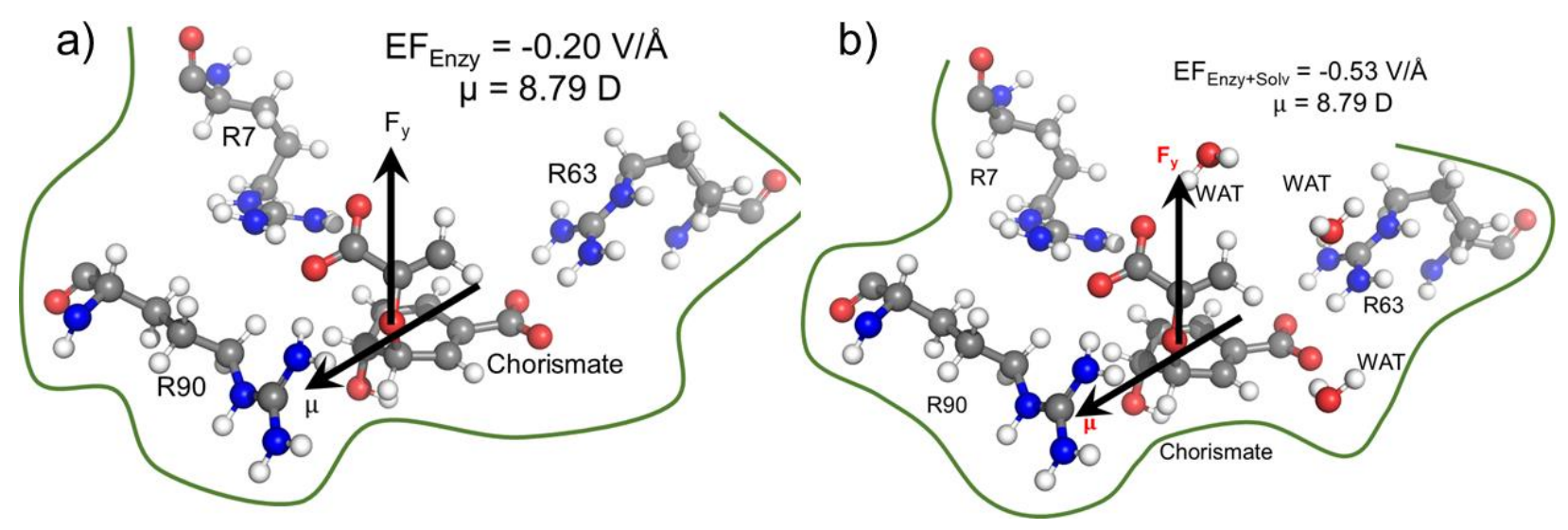

Figure 5. The Electric Field quantifications for Wild type Chorismate Mutase, a) Without solvent, b) with Solvent. We have used gaussian sign convention for the electric field and the dipole moments. 
To further justify the effect of electric field of enzyme on the catalysis, we performed the gasphase DFT calculations in the presence of external electric field of the same order of intensities exerted by the enzyme as calculated above. Figure 6 shows different TS barriers for the chorismate to prephenate conversion for different electric fields oriented along the y-axis (reaction axis) in positive and negative directions. The energy profiles at different EF clearly indicate that the electric field has a significant effect on the TS barrier.

a)

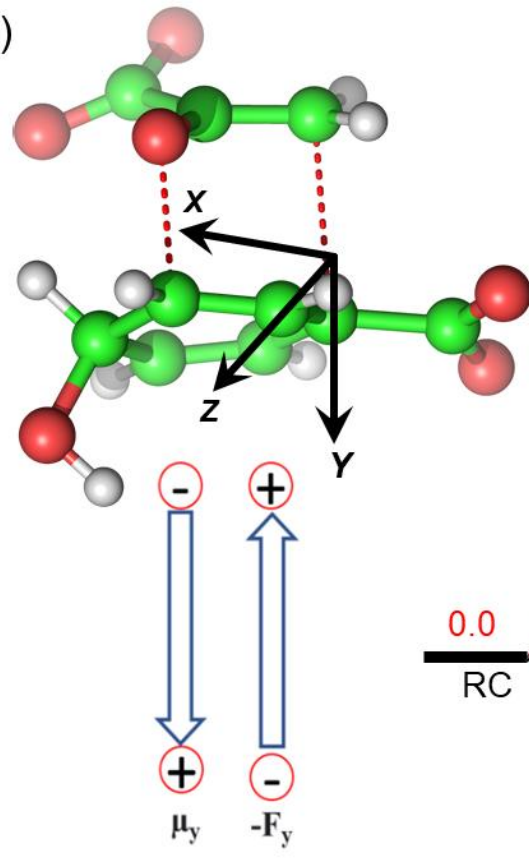

b) 65.78 
be more precise, we also calculated the effect of EEF on the dipole moments (see SI). It is quite apparent that the dipole moments of TS along the $\mathrm{Y}$-axis are positively signed which further increases towards positive scale on increasing the magnitude of EEF along the negative direction of $y$-axis. Hence, we can speculate that the application of EEF polarizes the TS in favourable manner and in turn decreases the TS barrier. The effect of EEF on dipole and hence on the activation energy, can also be validated by applying EEF in opposite direction. As can be seen

form Figure $6 \mathrm{~b}$, the reversal of the direction of external electric field with magnitude $0.2 \mathrm{~V} / \AA$ increased the TS barrier to $65.78 \mathrm{kcal} / \mathrm{mol}$, which is almost double of the observed in absence of EEF. The TS dipole moment due to reversal of the EEF is also decreased to 13.03 Debye, thereby, validating the effect of TS polarization of the reaction barrier. These results of the applications of external electric field that mimic the EF of enzymatic environment elucidate that the catalysis observed by the Bacillus subtilis chorismate mutase enzyme is electric field induced catalysis i.e., it uses its local electric field (LEF) to enhance the reaction million-folds. Furthermore, these results provide a qualitative agreement to the fact that protein environment of the enzyme produces a favorable electric field for the Claisen rearrangement reaction despite no covalent participation of protein residues.

\subsection{Electric Field: A new descriptor for Catalysis}

In the previous sections, we have seen that the enzymatic activity of the wild type CM is linearly dependent on the electric field produced by the enzyme. So, could we use electric field as a descriptor for enzymatic activity? We, therefore, focused to construe a correlation between the electric field of the several mutants of Chorismate mutase with their enzymatic activity. In this reference, initially we performed the MD simulations of R90G, R90A and R90K/C88S mutant complexes with TSA. The electric field of enzyme was calculated at a regular interval of 5ns 
projected along the ether-like $\mathrm{C}-\mathrm{O}$ bond vector of the TSA, and we reported the averaged value. The choice of these system was solely based on the fact that reactivity of these mutants is already known using experimental site-directed mutagenesis[43]. The electric field for the WT enzyme is found to be $\mathbf{- 0 . 3 2 7} \mathbf{V} / \mathbf{A}$ (Figure 6a), and it is opposite to the $\mathrm{C}-\mathrm{O}$ bond dipole vector illustrating that this EF would help the $\mathrm{C}-\mathrm{O}$ bond to get polarized and could favor the reaction which is in accord with the QM/MM calculations.
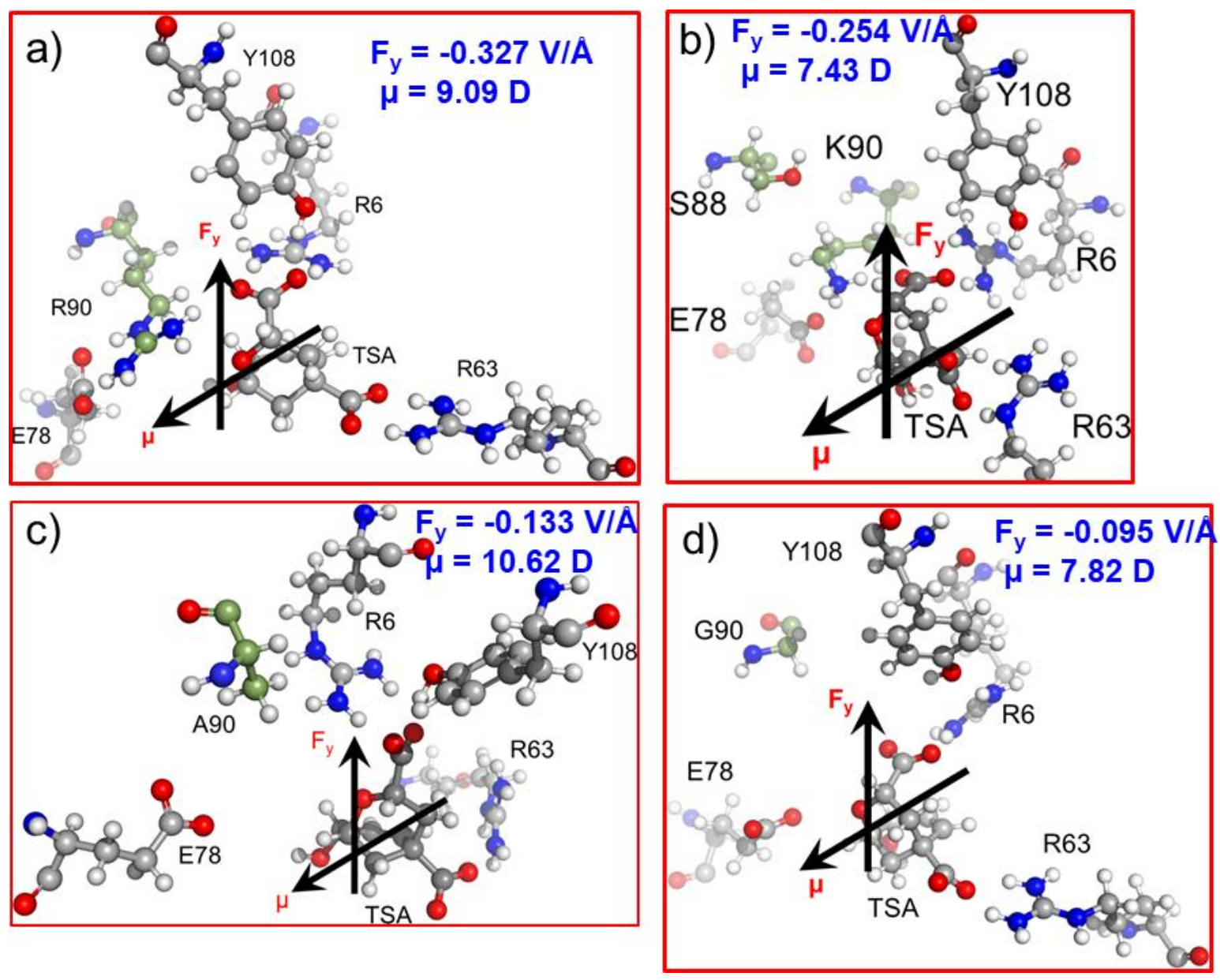

Figure 6. The dipole moments $\mu$ (in Debye) and LEFs ( $\mathrm{F}_{\mathrm{y}}$ in $\mathrm{V} / \AA$ ) along the $\mathrm{C}-\mathrm{O}$ bond axis of the TSA for a) WT CM \&, b) R90K/C88S, c) R90A and d) R90G mutants. The Gaussian convention is used to describe the direction of $\mathrm{EF}$ and dipole moments.

As shown in Figure 1 and Figure 2a, Arg90 forms a close and stable contact with the substrate and therefore, we anticipated a loss of EF due to the mutation of R90 with either Glycine or Alanine. 
In addition, the EF for R90A and R90G mutants were found to be $\mathbf{- 0 . 1 3 3 ~ V / \AA ~ a n d ~} \mathbf{- 0 . 0 9 5}$ V/Å, respectively which is approximately three times lower in magnitude than that of the wildtype enzyme (Figure 6c \& 6d). Interestingly, the experimental site directed mutagenesis for these mutations shows that the catalytic rate of mutant decreases by more than 5 orders of magnitude which is qualitatively in good agreement with the calculated EF of the mutant complex. To further confirm the role of electric field on catalysis R90 was strategically mutated to a charged residues K90. We also quantified EF for this R90K/C88S mutant and found that electric field increases significantly to $-0.254 \mathrm{~V} / \AA$ which is close to the WT complex (Figure 6b). Interestingly, the experimental determination of catalytic rate shows that the lost activity due to R90G and R90A mutations was restored. It clearly indicates that a quantification of relative electric field along the reaction axis can be used as a descriptor to qualify the relative reactivity of similar enzymes. However, a thorough benchmarking with different family of enzymes and its mutant may be needed for a generalized scope.

\section{Conclusion:}

In the present study, we have comprehensively studied the effect of local and external electric fields on the catalytic activity of Chorismate mutase using MD Simulation, hybrid QM/MM, EF quantification and application of EEF. The MD simulations of the Michaelis Complex (MC) and the Transition State Analogue (TSA) show that EF of enzyme CM stabilizes TSA, and it has evolved in such a way that EF produced by enzyme stabilizes the TS to reduces the activation energy. The DFT calculations in gas phase and enzyme environment prove the electric field produced by enzyme polarizes the transition state and increases its dipole moment which further reduces the TS barrier of the enzyme. The change in the dipole moment of TS demonstrates an electric field induced catalysis in Chorismate mutase. The quantification of EF for WT and mutants 
along the reactive axis shows a linear correlation between the enzymatic electric field and reactivity of enzyme, therefore, $\mathrm{EF}$ along the reactive axis could be a descriptor for the enzymatic activity. The EF as a descriptor for enzymatic activity has computational advantage over other activation energy as the quantification of EF is very simple and easy. However, this perception is limited to CM and its different variants, therefore, we believe that a rigorous benchmarking of a correlation between EF along the reaction axis and enzymatic activity using different enzymesubstrate is needed in future studies.

5. Acknowledgments: KDD acknowledge Department of Biotechnology, Govt. of India for Ramalingaswami Re-entry research grant (BT/RLF/Re-entry/10/2017).

Supporting Information: Supporting information contains the energy contribution calculated by MMPBSA calculations, dipole moments and energy values for different variants and coordinates for the QM and QM/MM optimized geometry of RC, TS and PC.

\section{References:}

1) S. Cocco, C. Feinauer, M. Figliuzzi, R. Monasson and M. Weigt, Rep. Prog. Phys.,2018, 81, 032601.

2) K. Davidsen, B. J. Olson, W. S. DeWitt III, J. Feng, E. Harkins, P. Bradley and F. A. Matsen IV, eLife, 2019, 8, e46935.

3) D. Repecka, V. Jauniskis, L. Karplus, E. Rembeza, J. Zrimec, S. Poviloniene, I Rokaitis, A. Laurynenas, W. Abuajwa, O. Savolainen, R. Meskeys, M. K. M. Engqvist and A. Zelezniak, Nat. Mach. Intell., 2021, 3, 324-333.

4) K. A. Reynolds, W. P. Russ, M. Socolich and R. Ranganathan, Methods Enzymol., 2013, $\mathbf{5 2 3}, 213-235$.

5) A.C. Anderson. Chemistry \& Biology, 2003,10,787-797.

6) R. Otten, R. A. P. Pádua, H. A. Bunzel, V. Nguyen, W. Pitsawong, M. Patterson, S. Sui, S. L. Perry, A. E. Cohen, D. Hilvert and D. Kern, Science, 2020, 370, 1442-1446. 
7) M.T.Reetz. Acc. Chem. Res. 2019, 52, 336-344.

8) F. H. Arnold, Acc. Chem. Res., 1998, 31, 125-131.

9) F. H. Arnold, Angew. Chem. Int. Ed., 2019, 58, 14420-14426.

10) M. T. Reetz, J. Am. Chem. Soc., 2013, 135, 12480-12496.

11) O. Keskin, A. Gursoy, B. Ma and R. Nussinov, Chemical Reviews, 2008, 108, 1225-1244.

12) P. Tuffery and P. Derreumax, J. R. Soc. Interface., 2012, 9, 20-33.

13) K. D. Dubey, R. K. Tiwari and R. P. Ojha, Curr Comput Aided Drug Des., 2013, 9, 518531.

14) R. E. Babine and S. L. Bender, Chem. Rev., 1997, 97, 1359-1472.

15) S. Strajbl, J. Florian and A. Warshel, J. Am. Chem. Soc., 2000, 122, 5354-5366.

16) J. Villa and A. Warshel, J. Phys. Chem. B., 2001, 105, 7887-7907.

17) A. Warshel, P. K. Sharma, M. Kato, Y. Xiang, H. Liu and M. H. M. Olsson, Chem. Rev., 2006, 106, 3210- 3235.

18) J. A. McCammon, B. R. Gelin and M. Karplus, Nature, 1977, 267, 585-590.

19) M. Karplus and J. A. McCammon, Nat. Struct. Biol., 2002, 9, 646-652.

20) K. D. Dubey and R. P. Ojha, in Nanoscience and Computational Chemistry, ed. A. G. Mercader, E. A. Castro and A. K. Haghi, CRC Press, Taylor and Francis Group, New Jersey, 1st edn, 2013, ch. 11, pp. 371-399.

21) H. M. Senn and W. Thiel, Angew. Chem., Int. Ed., 2009, 48, 1198-1229.

22) H. Hu, M. Elstner and J. Hermans, Proteins: Struct., Funct., Genet., 2003, 50, 451-463.

23) H. Lin and D. G. Truhlar, Theor. Chem. Acc., 2007, 117, 185-199.

24) U. Ryde, Curr. Opin. Chem. Biol., 2003, 7, 136-142.

25) T. Stuyver, J. Huang, D. Mallick, D. Danovich and S. Shaik, J. Comput. Chem. 2020, 41, 74-82.

26) T. Stuyver, J. Joy, D. Danovich and S. Shaik, in Effects of Electric Fields on Structure and Reactivity: New Horizons in Chemistry, ed. S. Shaik and T. Stuyver, Royal Society of Chemistry, London, 1st edn, 2021, ch. 6, pp. 195-224.

27) S. Shaik and T. Stuyver, in Effects of Electric Fields on Structure and Reactivity: New Horizons in Chemistry, ed. S. Shaik and T. Stuyver, Royal Society of Chemistry, London, 1st edn, 2021, ch. 1, pp.1-11. 
28) S. Shaik, D. Danovich, K. D. Dubey and T. Stuyver, in Effects of Electric Fields on Structure and Reactivity: New Horizons in Chemistry, ed. S. Shaik and T. Stuyver, Royal Society of Chemistry, London, 1st edn, 2021, ch. 2, pp. 12-70.

29) M. T. Blyth, M. L. Coote, in Effects of Electric Fields on Structure and Reactivity: New Horizons in Chemistry, ed. S. Shaik and T. Stuyver, Royal Society of Chemistry, London, 1st edn, 2021, ch. 4, pp. 119-146.

30) S. Sowlati-Hashjin, N. Karttunen, C. F. Matta, in Effects of Electric Fields on Structure and Reactivity: New Horizons in Chemistry, ed. S. Shaik and T. Stuyver, Royal Society of Chemistry, London, 1st edn, 2021, ch. 7, pp. 225-262.

31) N. J. English, J. Mol. Liquids, 2021, DOI: https://doi.org/10.1016/j.molliq.2021.116949, In press.

32) N. J. English, in Effects of Electric Fields on Structure and Reactivity: New Horizons in Chemistry, ed. S. Shaik and T. Stuyver, Royal Society of Chemistry, London, 1st edn, 2021, ch. 8, pp. 225-262.

33) S. Shaik, D. Mandal and R. Ramanan, Nat. Chem., 2016, 8, 1091-1098.

34) S. Shaik, R. Ramanan, D. Danovich and D. Mandal, Chem. Soc. Rev., 2018, 47, 5125-5145.

35) S. Ciampi, N. Darwish, H. M. Aitken, I. Diez-Pérez and M. L. Coote. Chem. Soc. Rev. 2018, 47, 5146-5164.

36) P. R. Andrews, G. D. Smith and I. G. Young, Biochemistry, 1973, 12, 3492-3498.

37) S. D. Copley and J. R. Knowles, J. Am. Chem. Soc., 1987, 109, 5008-5013.

38) P. Kast, M. Asif-Ullah and D. Hilvert, Tetrahedron Lett., 1996, 37, 2691-2694.

39) P. Kast, M. Asif-Ullah, M., N. Jiang and D. Hilvert, Proc. Natl. Acad. Sci., 1996, 93, 50435048.

40) S. Martí, J. Andrés, V. Moliner, E. Silla, I. Tuñón, J. Bertrán and M. J. Field, J. Am. Chem. Soc., 2001, 123, 1709-1712.

41) S. Martí, J. Andrés, V. Moliner, E. Silla, I. Tuñón and J. Bertrán, Chem. Eur. J., 2003, 9, 984-991.

42) S. Martí, J. Andrés, V. Moliner, E. Silla, I. Tuñón and J. Bertrán, J. Am. Chem. Soc., 2004, 126, 311-319.

43) A. Mandal and D. Hilvert, J. Am. Chem. Soc., 2003, 125, 5598-5599.

44) A. Aemissegger, B. Jaun and D. Hilvert, J. Org. Chem., 2002, 67, 6725-6730. 
45) Y. M. Chook, H. Ke and W. N. Lipscomb, Proc. Natl. Acad. Sci., 1993, 90, 8600-8603.

46) W. L. Jorgensen, J. Chandrasekhar, J. D. Madura, R. W. Impey and M. L. Klein, J. Chem. Phys., 1983, 79, 926-935.

47) O. Trott and A. J. Olson, J. Comput. Chem., 2010, 31, 455-461.

48) J. A. Izaguirre, D. P. Catarello, J. M. Wozniak and R. D. Skeel, J. Chem. Phys., 2001, 114, 2090-2098.

49) H. J. C. Berendsen, J. P. M. Postma, W. F. van Gunsteren, A, DiNola and J. R. Haak, J. Chem. Phys., 1984, 81, 3684-3690.

50) J. P. Ryckaert, G. Ciccotti and H. J. C. Berendsen, J. Comput. Phys., 1977, 23, 327-341.

51) T. Darden, D. York and L. Pederson, J. Chem. Phys., 1993, 98, 10089-10092.

52) R. S. Ferrer, A. W. Götz, D. Poole, S. L. Grand and R. C. Walker, J. Chem. Theory Comput., 2013, 9, 3878-3888.

53) P. Sherwood, A. H. de Vries, M. F. Guest, G. Schreckenbach, C. R. A. Catlow, S. A. French, A. A. Sokol, S. T. Bromley, W. Thiel, A. J. Turner, S. Billeter, F. Terstegen, S. Thiel, J. Kendrick, S. C. Rogers, J. Casci, M. Watson, F. King, E. Karlsen, M. Sjøvoll, A. Fahmi, A. Schäfer and C. Lennartz, J. Mol. Struct.: THEOCHEM, 2003, 632, 1-28.

54) S. Metz, J. Kästner, A. A. Sokol, T. W. Keal and P. Sherwood, Comput. Mol. Sci., 2014, 4, 101-110.

55) R. Ahlrichs, M. Bär, M. Häser, H. Horn and C. Kölmel, Chem. Phys. Lett., 1989, 162, 165-169.

56) W. Smith and T. R. Forester, J. Mol. Graphics, 1996, 14, 136-141.

57) J. A. Maier, C. Martinez, K. Kasavajhala, L. Wickstrom, K. E. Hauser and C. Simmerling, J. Chem. Theory Comput., 2015, 11, 3696-371.

58) A. D. Becke, J. Chem. Phys., 1993, 98, 5648-5652.

59) M. J. Frisch, G. W. Trucks, H. B. Schlegel, G. E. Scuseria, M. A. Robb, J. R. Cheeseman, G. Scalmani, V. Barone, B. Mennucci, G. A. Petersson, H. Nakatsuji, M. Caricato, X. Li, H. P. Hratchian, A. F. Izmaylov, J. Bloino, G. Zheng, J. L. Sonnenberg, M. Hada, M. Ehara, K. Toyota, R. Fukuda, J. Hasegawa, M. Ishida, T. Nakajima, Y. Honda, O. Kitao, H. Nakai, T. Vreven, J. A. Montgomery, Jr., J. E. Peralta, F. Ogliaro, M. Bearpark, J. J. Heyd, E. Brothers, K. N. Kudin, V. N. Staroverov, T. Keith, R. Kobayashi, J. Normand, K. Raghavachari, A. Rendell, J. C. Burant, S. S. Iyengar, J. Tomasi, M. Cossi, N. Rega, J. M. 
Millam, M. Klene, J. E. Knox, J. B. Cross, V. Bakken, C. Adamo, J. Jaramillo, R. Gomperts, R. E. Stratmann, O. Yazyev, A. J. Austin, R. Cammi, C. Pomelli, J. W. Ochterski, R. L. Martin, K. Morokuma, V. G. Zakrzewski, G. A. Voth, P. Salvador, J. J. Dannenberg, S. Dapprich, A. D. Daniels, O. Farkas, J. B. Foresman, J. V. Ortiz, J. Cioslowski, and D. J. Fox, Gaussian 09, Revision D.01, Gaussian, Inc., Wallingford CT, 2013. 